\title{
Antarctic subglacial drilling rig: Part II. Ice and Bedrock Electromechanical Drill (IBED) - CORRIGENDUM
}

\section{Corrigendum}

Cite this article: Talalay P et al. (2021) Antarctic subglacial drilling rig: Part II. Ice and Bedrock Electromechanical Drill (IBED) CORRIGENDUM. Annals of Glaciology 62(84), 23-23. https://doi.org/10.1017/aog.2020.53

First published online: 27 July 2020
Pavel Talalay ${ }^{1}$ (I) Xingchen $\mathrm{Li}^{1}$, Nan Zhang ${ }^{1}$, Xiaopeng Fan ${ }^{1}$, Youhong Sun ${ }^{1,2}$, Pinlu Cao ${ }^{1}$, Rusheng Wang ${ }^{1}$, Yang Yang ${ }^{1}$, Yongwen $\mathrm{Liu}^{1}$, Yunchen $\mathrm{Liu}^{1}$, Wei $\mathrm{Wu}^{1}$, Cheng Yang ${ }^{1}$, Jialin Hong ${ }^{1}$, Da Gong ${ }^{1}$, Han Zhang ${ }^{1}$, Xiao Li $^{1}$, Yunwang Chen ${ }^{1}$, An Liu ${ }^{1}$ and Yazhou Li ${ }^{1}$

${ }^{1}$ Polar Research Center, Jilin University, Changchun, China and ${ }^{2}$ China University of Geosciences, Beijing, China https://doi.org/10.1017/aog.2020.38. Published by Cambridge University Press, 11 June 2020 The title of this article was amended to bring it in line with other articles in the series. 